\title{
A Hybrid Two-Phase Machine Learning Model for Early COVID-19 Diagnosis Prediction
}

\author{
Ahmed S. Salama \\ Computers and Information Systems Department \\ Sadat Academy for Management Sciences \\ Cairo, Egypt
}

\begin{abstract}
Across the history, COVID-19 pandemic is considered one of the deadliest diseases that harvested more than one million souls and left thousands of patients with damaged fibrotic lungs that physicians called post COVID syndrome. The main aim of this study is to propose a hybrid two-phase machine learning model to early diagnose COVID-19 based on available laboratory tests results, clinical symptoms, CT results, and demographic data in case of the difficulty of applying or absence of PCR test. The proposed model employs unsupervised learning Scalable Expectation Maximization (SEM) soft clustering mining model in the first phase to identify the most relevant identifying clusters characteristics for the disease grades, and in phase two the proposed model applies two proposed supervised learning classification mining models which are Association Rules (AR) based on improved Apriori algorithm, and Multilayer Perceptron(MLP) Multiclass Artificial Neural Network (ANN) to predict the COVID-19 disease diagnosis. The implemented proposed ML hybrid COVID-19 prediction model has successfully classified COVID-19 patients into positive mild, positive severe patients and discriminated between COVID-19 and Influenza patients/normal cases (COVID-19 negative) with an overall accuracy of $97.3 \%$, a sensitivity $96 \%$, and specificity $98 \%$. It outperforms other reviewed state-of-the art COVID-19 diagnosis prediction models.
\end{abstract}

\section{General Terms}

Artificial Intelligence, Machine Learning, COVID-19

\section{Keywords}

Covid-19; Hybrid Model; Machine Learning; Soft Clustering; Association Rules; MLP Multiclass Artificial Neural Networks

\section{INTRODUCTION}

In the city of Wuhan, China in December 2019, pneumonia of unidentified aetiologia (novel coronavirus) was detected with its first death recorded on January 10, 2020, has turned into a pandemic, and labeled by the World Health Organization (WHO) as COVID-19 (Coronavirus 2019) [1]. Latest research has shown the promising opportunities of $\mathrm{AI}$ and ML techniques and algorithms for different pandemic breakouts. They help health professionals in different transmissible diseases (SARS, EBOLA, HIV, COVID 19) [2,3,4] and outbreak of diseases that are not communicable (Cancer, Diabetic, Heart, and Stroke) [5,6]. Early and quick identification of diseases, whether they are contagious or noncontagious, is a vital activity that gives health experts enough time to save lives early and lowers medical cost and disease outbreak $[7,8]$. The paper is organized as follows: section 2 presents a literature review of the related work. Section 3 introduces the proposed two-phase hybrid machine learning based model parameters explanation, architecture, proposed AR, and MLP ANN algorithms for COVID-19 diagnosis and prediction. Section 4 presents the implementation issues and the experimental results of the proposed hybrid model. Finally, section 5 introduces conclusions and a look forward for the expected future work in this domain.

\section{RELATED WORK}

Several studies and research were done on using AI and machine learning techniques to diagnose COVID-19 disease. This section introduces a review for some of them.

Javor et al. [9] introduced a new deep learning-based machine learning classification (ML) with a simplified programming approach, with an open-source dataset consisting of 6,868 CT images from 418 patients. Receiver Operating Characteristics (ROC) analysis was used to calculate Diagnostic performance metrics Rule-in and rule out thresholds were determined and checked. At the rule-in operating point, sensitivity and specificity were $84.4 \%$ and $93.3 \%$. At the rule-out threshold, sensitivity $(100 \%)$ and specificity $(60 \%)$. Goodman-Meza et al. [10] aimed at designing and testing the COVID-19 inpatient diagnostic machine learning algorithm. The proposed algorithm has been used as a screening method based on basic demographic and laboratory features. For the final diagnostic classification, seven machine learning models were tested and employed as a combination. The model has reached 0.93 sensitivity and 0.64 specificity. An et al. [11] introduced a diagnostic model for COVID-19 diagnosis based on machine learning techniques that include the least absolute shrinkage and selection operator (LASSO), linear support vector machine (SVM), SVM with radial basis function kernel, random forest (RF), and k-nearest neighbors were applied. In mortality prediction, LASSO and linear SVM has achieved high sensitivities (90.7\% and $92.0 \%$ respectively) and specificities (91.4\% and $91.8 \%$ respectively). Khanday et al. [12] proposed a four-class approach which classified textual clinical reports using traditional and hybrid machine learning algorithms. The features were given to classifiers for conventional and hybrid machine learning. By having 96.2 percent accuracy testing, logistic regression and multinomial Naïve Bays have shown better results than other ML algorithms. For greater accuracy recurrent neural network can be employed. Banerjee et al. [13] presented four machinestudy models that have been tested on the basis of blood tests to initially screen suspect COVID-19 cases. The models used include random forest (RF), artificial neural network (ANN), linear regression (LR) and generalized regularized Lassoelastic network (GLMNET). The models have reached $81-$ $87 \%$ of accuracy; $43-65 \%$ of sensitivity and $81-91 \%$ of specificity. Bao et al. [14] introduced models for the early 
detection of COVID-19 based on routine blood tests were investigated by random forest (RF) and the support vector machine (SVM). Three classification activities have been carried out (moderate vs viral, severe vs. viral and severe vs. moderate). Up to 15 blood characteristics were chosen to train the models. The best performance of the SVM-based classifier was $84 \%$ accuracy, $88 \%$ sensitivity, $80 \%$ specificity and $92 \%$ precision. Barbosa et al. [15] built a cheap COVID-19 blood sample detection system, using several ML classifications, including SVM, random forest (RF) and Bayesian networks $(\mathrm{BN})$. In order to further minimize the expense and length of blood tests, features of training and testing of their models were reduced to 24 . With $95.159 \%$ of the overall accuracy, sensitivity of $96.8 \%$, precision of $93.8 \%$, and specificity of $93.6 \%$, the result achieved high classification efficiency. Experiments showed that $\mathrm{BN}$ performed better in comparison to other models. Nan et al. [16] used five types of classification models to identify the most powerful early diagnostic model for the COVID-19 infection by using: logistic regression (LR), support vector machine (SVM), decision tree(DT), random forest (RF) and deep learning neural network(DNN). The best performance was achieved by the LR classification model among the five classifiers with $91 \%$ accuracy, $87 \%$ sensitivity and $95 \%$ specificity. Bayat et al. [17] established a predictive random forest (RF) model of COVID-19 by combining clinical signs with common lab tests. The model was trained with $40-54$ parameters, which resulted in $88.3 \%$ accuracy, $83.4 \%$ sensitivity and $89.8 \%$ specificity. Zoabi Y. et al. [18] proposed a machine-learning approach in which predictions were made using a gradientboosting machine model built with decision-tree baselearners. The model predicted with high precision COVID-19 test results using only eight binary characteristics: sex, age 60 years, documented contact with the infected person and five initial symptoms.

\section{THE PROPOSED HYBRID TWO- PHASE MACHINE LEARNING BASED MODEL FOR COVID-19 DISEASE DIAGNOSIS}

In this section, the proposed hybrid model input and output parameters, architecture, and proposed used mining models' algorithms are introduced.

\subsection{The Proposed Hybrid Model Input and Output Parameters}

Based on a review of practice, chest consultants' opinions surveys, and empirical literature, the proposed model empowers six important categories of input parameters (20 features) that are used to diagnose Covid-19: A complete blood count (CBC) parameters, immunology parameter, chemistry parameters, clinical symptoms parameters, CT results parameter and demographic data parameters. The $\mathrm{CBC}$, immunology, and chemistry parameters belong to generic category called laboratory tests. The CT results are represented using a common CO-RADS: a categorical CT assessment scheme for patients with suspected COVID- 19 [19]. CO-RADS is a CT-based system that is used to assess the suspicion of pulmonary involvement in COVID-19. For a patient diagnosis with COVID-19, CO-RADS needs to be accompanied with other data, such as laboratory test results, clinical findings, and type and duration of symptoms. That is why this proposed ML hybrid model used all these types of categories for predicting accurate COVID-19 diagnosis. These categorized input parameters with their descriptions are shown in Table 1. In addition, main predicted output parameter for the proposed model which is Covid-19 Diagnosis with its description is shown in Table 2. Negative means that the investigated person either normal or infected with other disease that is not compatible with COVID-19 such as Influenza, Positive Mild means that the patient is COVID19 patient with mild lab test results, clinical symptoms, and CT result (CO-RADS 4), and Positive Severe means that the COVID-19 patient has typical features for pulmonary involvement of COVID-19 (CO-RADS 4 or 5) and supportive severe lab test results, and clinical symptoms. In this case, the patient may need noninvasive (CPAP), or invasive ventilation. The proposed hybrid model parameters for predicting Covid19 diagnosis accompanied with normal range values, domain values and types are presented in Table 3 .

Table 1. Categorized Covid-19 Model Diagnostic Input Parameters

\begin{tabular}{|c|c|c|}
\hline \multicolumn{3}{|c|}{ 1. CBC Parameters (Laboratory Tests) } \\
\hline No & Parameter Name & Description \\
\hline 1 & $\begin{array}{l}\text { Haemoglobin } \\
\text { (HB) }\end{array}$ & $\begin{array}{l}\text { A protein found in the red } \\
\text { blood cells that carries } \\
\text { oxygen in your body }\end{array}$ \\
\hline 2 & $\begin{array}{l}\text { Red Blood Cell } \\
\text { Count (RBC) }\end{array}$ & $\begin{array}{l}\text { How many red blood cells } \\
\text { (RBCs) a person has }\end{array}$ \\
\hline 3 & $\begin{array}{l}\text { Platelet Count } \\
\text { (PC) }\end{array}$ & $\begin{array}{l}\text { The average number } \\
\text { of platelets in the blood. }\end{array}$ \\
\hline 4 & $\begin{array}{l}\text { Total } \\
\text { leucocyte count } \\
\text { (T.L.C.) }\end{array}$ & $\begin{array}{l}\text { The number of leucocytes in } \\
\text { the body }\end{array}$ \\
\hline 5 & Lymphocytes\% & $\begin{array}{c}\text { Measures } \\
\text { percentage of lymphocytes: } \\
\text { white blood cells (immune } \\
\text { cells) }\end{array}$ \\
\hline \multicolumn{3}{|c|}{ 2. Immunology (Laboratory Test) } \\
\hline No & Parameter Name & Description \\
\hline 1 & $\begin{array}{l}\text { Ferritin in serum } \\
\text { (F) }\end{array}$ & $\begin{array}{c}\text { inflammatory marker: } \\
\text { a marker of cellular damage }\end{array}$ \\
\hline \multicolumn{3}{|c|}{ 3. Chemistry (Laboratory Tests) } \\
\hline No & Parameter Name & Description \\
\hline 1 & $\begin{array}{l}\text { level of lactate } \\
\text { dehydrogenase } \\
\text { (LDH) serum }\end{array}$ & $\begin{array}{c}\text { High LDH blood or fluid } \\
\text { means certain tissues in your } \\
\text { body have been damaged by } \\
\text { disease }\end{array}$ \\
\hline 2 & $\begin{array}{l}\text { C-reactive protein } \\
(\mathrm{CRP})\end{array}$ & $\begin{array}{l}\text { a blood test marker for } \\
\text { inflammation in the body }\end{array}$ \\
\hline 3 & D Dimer & $\begin{array}{l}\text { a protein fragment (small } \\
\text { piece) that has made when a } \\
\text { blood clot dissolves in your } \\
\text { body }\end{array}$ \\
\hline 4. & ults & \\
\hline
\end{tabular}




\begin{tabular}{|c|c|c|}
\hline No & Parameter Name & Description \\
\hline 1 & $\begin{array}{l}\text { CT COVID-19 } \\
\text { grade }\end{array}$ & $\begin{array}{l}\text { The level of suspicion of } \\
\text { COVID-19 infection } \\
\text { including the severity and } \\
\text { stage of the disease graded } \\
\text { from very low (negative) or } \\
\text { CO-RADS } 1 \text { up to very high } \\
\text { or CO-RADS } 5\end{array}$ \\
\hline \multicolumn{3}{|c|}{ 5. Clinical Symptoms } \\
\hline No & $\begin{array}{c}\text { Parameter } \\
\text { Name }\end{array}$ & Description \\
\hline 1 & Body Temp (BT) & $\begin{array}{c}\text { a measure of } \\
\text { your body's ability to make } \\
\text { and get rid of heat }\end{array}$ \\
\hline 2 & Headache & a continuous pain in the head \\
\hline 3 & Body aches & Body pain \\
\hline 4 & $\begin{array}{l}\text { Loss of Smell \& } \\
\text { Taste senses } \\
\text { (anosmia and } \\
\text { ageusia) }\end{array}$ & $\begin{array}{l}\text { complete loss or absence of } \\
\text { smell and taste }\end{array}$ \\
\hline 5 & Diarrhea & $\begin{array}{c}\text { loose, watery stools (bowel } \\
\text { movements) }\end{array}$ \\
\hline 6 & Dyspnea & Shortness of breath \\
\hline 7 & $\begin{array}{l}\text { Influenza Like } \\
\text { Symptoms }\end{array}$ & $\begin{array}{l}\text { A group of symptoms that } \\
\text { are similar to those caused } \\
\text { by the influenza (flu) virus }\end{array}$ \\
\hline 8 & Other Symptoms & $\begin{array}{l}\text { Recorded Covid-19 other } \\
\text { rare symptoms include red } \\
\text { eye, skin or CNS } \\
\text { manifestations, stomachache }\end{array}$ \\
\hline \multicolumn{3}{|c|}{ 6. Demographic Data } \\
\hline No & Parameter Name & Description \\
\hline 1 & gender & $\begin{array}{c}\text { Person Sex Type (Male or } \\
\text { Female) }\end{array}$ \\
\hline 2 & Age & $\begin{array}{l}\text { The length of time that a } \\
\text { person has lived }\end{array}$ \\
\hline
\end{tabular}

Table 2. Covid-19 Predicted Output Parameter

\begin{tabular}{|c|c|c|}
\hline No & Parameter Name & Description \\
\hline 1 & Diagnosis & $\begin{array}{c}\text { Decision whether there is } \\
\text { Covid-19 from its signs and } \\
\text { symptoms }\end{array}$ \\
\hline
\end{tabular}

Table 3. Categorized Covid-19 Diagnostic Model Parameters Normal Ranges, Domain, and Types

\begin{tabular}{|c|c|c|c|}
\hline No & $\begin{array}{l}\text { Parameter } \\
\text { Name }\end{array}$ & Normal Range & $\begin{array}{l}\text { Parameter } \\
\text { Type }\end{array}$ \\
\hline 1 & HB & $11.5-16 \mathrm{~g} / \mathrm{dL}$ & Continuous \\
\hline 2 & $\mathrm{RBC}$ & $3.8-5.4 * 10^{6} / \mathrm{uL}$ & Continuous \\
\hline 3 & $\begin{array}{c}\text { Platelet } \\
\text { Count (PC) }\end{array}$ & $150-350 * 10^{3} / \mathrm{uL}$ & Continuous \\
\hline 4 & (T.L.C.) & $4-11 * 10^{3} / \mathrm{uL}$ & Continuous \\
\hline 5 & $\begin{array}{c}\text { Lymphocytes } \\
\%\end{array}$ & $20-45 \%$ & Continuous \\
\hline 6 & $\begin{array}{l}\text { Ferritin in } \\
\text { serum }(F)\end{array}$ & $10-120 \mathrm{ng} / \mathrm{mL}$ & Continuous \\
\hline 7 & $\begin{array}{l}(\mathrm{LDH}) \\
\text { serum }\end{array}$ & $0-247 \mathrm{U} / \mathrm{L}$ & Continuous \\
\hline 8 & (CRP) & $<5 \mathrm{mg} / \mathrm{L}$ & Continuous \\
\hline 9 & D Dimer & $<0.5 \mu \mathrm{g} \mathrm{FEU} / \mathrm{ml}$ & Continuous \\
\hline 10 & $\begin{array}{l}\text { Body Temp } \\
\text { (BT) }\end{array}$ & $\begin{array}{c}\text { Normal: } 36.1^{\circ} \mathrm{C} \text { to } \\
37.2^{\circ} \mathrm{C} \\
\text { Fever: }>37.5 \text { to } \\
38.3^{\circ} \mathrm{C} \\
\text { High Fever: }>40.0 \\
\text { or } 41.0^{\circ} \mathrm{C}\end{array}$ & Continuous \\
\hline No & $\begin{array}{l}\text { Parameter } \\
\text { Name }\end{array}$ & Domain & $\begin{array}{l}\text { Parameter } \\
\text { Type }\end{array}$ \\
\hline 11 & Headache & No (0), Yes (1) & Discrete \\
\hline 12 & Body aches & No (0), Yes (1) & Discrete \\
\hline 13 & $\begin{array}{c}\text { Loss of Smell } \\
\text { \& Taste } \\
\text { senses }\end{array}$ & No (0), Yes (1) & Discrete \\
\hline 14 & Diarrhea & No (0), Yes (1) & Discrete \\
\hline 15 & Dyspnea & No (0), Yes (1) & Discrete \\
\hline 16 & $\begin{array}{l}\text { Influenza } \\
\text { Like } \\
\text { Symptoms }\end{array}$ & No (0), Yes (1) & Discrete \\
\hline 17 & $\begin{array}{c}\text { Other } \\
\text { Symptoms }\end{array}$ & No (0), Yes (1) & Discrete \\
\hline 18 & Gender & $\begin{array}{l}\text { Male (1), } \\
\text { Female(2) }\end{array}$ & Discrete \\
\hline 19 & Age & $1-115$ & Discrete \\
\hline
\end{tabular}




\begin{tabular}{|c|c|l|l|}
\hline 20 & $\begin{array}{c}\text { CT COVID- } \\
19 \text { grade }\end{array}$ & $\begin{array}{l}\text { (Negative) CO- } \\
\text { RADS 1, 2, 3 1 } \\
\text { (Positive Mild) } \\
\text { CO-RADS 4 2 } \\
\text { (Positive Severe) } \\
\text { CO-RADS 5 }\end{array}$ & Discrete \\
\hline 21 & Diagnosis & $\begin{array}{c}\text { 0 Negative } \\
\text { (COVID-19 } \\
\text { Absence) } \\
\text { 1 Positive Mild } \\
\text { 2 Positive Severe }\end{array}$ & Discrete \\
\hline
\end{tabular}

\subsection{The Proposed Hybrid Two-Phase Machine Learning Model for Predicting COVID-19 Diagnosis Architecture}

The main components of the proposed hybrid model for predicting COVID-19 diagnosis architecture, and the two important implemented phases of the proposed unsupervised and supervised learning process to reach an accurate prediction for COVID-19 diagnosis and their outputs are presented in Fig. 1. Data cleaning, selection, and preparation operations involve removing of patients' medical data records that have missed values, redundancies, and noise or outliers. Next, a selection of the candidate parameters (features) for the mining process is performed. Finally, a data preparation process is performed that includes converting nominal attributes values to numerical values using integer encoding (Table 3). Last step is to convert the refined medical records dataset into a relational DB that is ready for mining. The Scalable Expectation Maximization (EM) algorithm as a soft clustering method [20], a Multilayer Perceptron (MLP) multiclass artificial neural network classifier and predictor mining model, and Association Rules (AR) based on improved Apriori algorithm classifier and predictor mining model are applied in this proposed model.

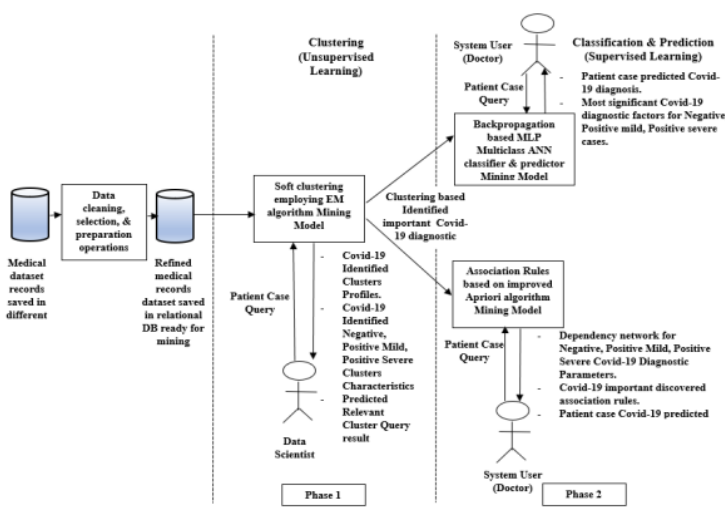

Fig. 1: Architecture of the Proposed Hybrid Two-Phase Machine Learning Model for COVID-19 Diagnosis Prediction

\subsection{Proposed Architecture and Algorithm for Back Propagation based MLP Multiclass ANN Mining Model}

The proposed architecture of the Back Propagation based MLP multiclass ANN mining model for Predicting COVID19 Diagnosis is shown in Fig. 2. The architecture of the proposed ANN consists of the input layer which contains a vector of twenty processing elements (PEs) which are the important identified COVID-19 diagnostic input parameters during clustering phase. Where $\mathrm{x}_{1} . . \mathrm{x}_{20}$ represent the input parameters for the ANN classified into 6 main categories: CBC parameters, Immunology parameter, Chemistry parameters, CT results, Clinical Symptoms, and Demographic data Parameter. The ANN also consists of one hidden layer with a vector of $n$ PEs. $h_{1} . . h_{n}$ represent the $n$ processing elements PEs in the hidden layer where the initial number of PEs in the hidden layer is $\approx 31$ according to formula (4), and output layer with three PEs representing the three classes (states) of the COVID-19 predicted Diagnosis parameter, which are 0 Negative, 1 Positive Mild, and 2 Positive Severe. Listing of the input and output parameters of the proposed ANN model is explained previously in Table 1, and Table 2 section 4.1. Each $\mathrm{W}_{\mathrm{ij}}$ represents the weight of the connection from the ith input $P E x_{i}$ in the input layer to the jth hidden PE $h_{j}$ in the hidden layer, and every $\mathrm{w}_{\mathrm{jk}}$ represents weight of connection from the jth hidden $P E h_{j}$ in the hidden layer to the kth output $P E y_{k}$ in the output layer.

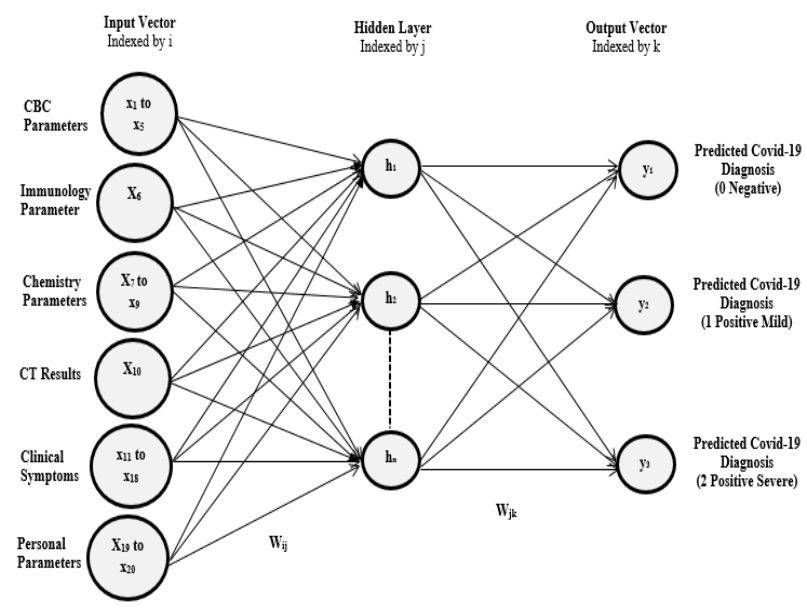

Fig. 2: The Back Propagation Based MLP ANN Based Mining Model for COVID-19 Diagnosis Prediction

Each PE $\left(h_{j}\right)$ in the hidden layer or $\left(y_{k}\right)$ in the output layer will do summation to combine and modify the inputs from the previous layer using the following equation:

$m_{j}=\sum_{i=1}^{n} x_{i} w_{i j}+b_{j}$

where $m_{j}$ is the net input to $h_{j}$ in hidden or to $y_{k}$ in output layer, $x_{i}$ is the input to $P_{j}$ (or outputs of previous layer), $i$ is the number of $\mathrm{PE}$ in previous layer, $\mathrm{n}$ is the number of inputs and $b_{j}$ is the bias associated with $P E_{j}$. This weighted sum, then is passed to the activation function which is a hyperbolic tangent function (tanh) in the hidden layer PEs, whereas output PEs use a sigmoid function for activation. These used activation functions are as follows:

(sigmoid function) $f(x)=\frac{1}{1+e^{-x}}$

(hyperbolic tangent function) $f(x)=\frac{e^{x}-e^{-x}}{e^{x}+e^{-x}}$

where $\mathrm{x}$ is the input and $\mathrm{f}$ is the output.

The formula used to determine the initial number of PEs in the hidden layer $\left(\mathrm{h}_{\mathrm{n}}\right)$ is as follows:

$$
h n=r * \sqrt{i * o}
$$

where $r$ is the hidden node ratio (default value is 4.0), $i$ is the total input PEs, and $o$ is the total output PEs. 
Algorithm 1: Proposed Backpropagation based MLP ANN Mining Model Algorithm for COVID-19 Diagnosis

Prediction

1. TP: True Positive; TN: True Negative; FP: False Positive; FN: False Negative

2. D: Selected patients' dataset for identified COVID-19 diagnostic input parameters during clustering phase.

3. $\mathrm{X}$ : The total number of cases COVID-19 diagnosis values to be predicted by the model.

4. $\mathrm{m}$ : It takes value from 1 to 3 representing three diagnosis classes (Negative, Positive Mild, Positive Severe).

5. Initialize all network weights and thresholds with random values between -1 to 1 .

6. Initialize macc $=0.0 / /$ Initializes ANN mining model accuracy with 0 .

7. Calculate $h n / /$ initial number of PEs in the hidden layer using formula (4)

8. Determine percentage of the training and testing data of D.

9. Repeat the following for the training dataset:

10. Calculate the weighted sum for each hidden PE $\left(h_{j}\right)$ in the hidden layer using formula (1).

11. Apply hidden layer activation function (3) to the calculated weighted sum.

12. Calculate the weighted sum for each output PE $\left(\mathrm{y}_{\mathrm{k}}\right)$ in the output layer using formula (1).

13. Apply output layer activation function

(2) to the calculated weighted sum.

14. Calculate the difference between the predicted and actual value for the output PEs. (error)

15. The gradients of the errors for the neurons in the output layer are calculated.

16. The gradients for the weights between the hidden layer and the output layer are updated.

17. The gradients of the errors for the neurons in the hidden layer are calculated.

18. The gradients of the weights between the input layer and the hidden layer are updated.

19. Update the weights of all the connections based on the gradients of the weights using this formula:

$$
w i j=w i j+\eta \cdot \Delta w i j
$$

where $\eta$ is the learning rate.

20. Apply the learned ANN mining model on the testing dataset.

21. Calculate nmacc as follows: // nmacc is calculated new ANN mining model overall accuracy derived from 3-class confusion matrix

nmacc $=$

$\frac{\sum_{m=1}^{3} T P_{m}+\sum_{m=1}^{3} T N_{m}}{\sum_{m=1}^{3} T P_{m}+\sum_{m=1}^{3} T N_{m}+\sum_{m=1}^{3} F P_{m}+\sum_{m=1}^{3} F N_{m}}$

(6)

22. If nmacc >mcc Then

Assign nmacc to macc

Go to step 9

23. Endi

24. Stop // terminate training when the mining model accuracy no longer increases.

\subsection{Proposed Improved Apriori Algorithm based Association Rules (AR) Mining Model}

In this section, an improved Apriori algorithm for Association Rules mining model is presented. Improving the Apriori algorithm is done by defining the low minimum support, high minimum confidence, and minimum importance percentage to generate important COVID-19 diagnosis prediction association rules.

\section{Algorithm 2: Proposed Association Rules mining model} Algorithm based on Improved Apriori algorithm

1. D: selected patients' dataset for identified COVID-19 diagnostic input parameters during clustering phase.

2. Join step: $\mathrm{C}$ is generated by joining $\mathrm{Lx}^{-1}$ with itself.

3. Prune step: Any $\left(x^{-1}\right.$ itemset $)$ that is not frequent remove it.

4. C: Candidate itemset of size $x$.

5. L: Frequent itemset of size $x$.

6. c : Candidate set in $\mathrm{C}$.

7. count: frequency support counter of candidate set $\mathrm{c}$.

8. cv: Confidence value.

9. $\varepsilon$ : Support threshold // minimum support count

10. min_conf: Minimum confidence percentage or minimum probability percentage

11. min_imp: Minimum importance percentage or minimum lift percentage

12. ARS: Set of generated Association Rules.

13. A: Association Rules that their confidence values $\geq$ min_conf and importance value $\geq$ min imp.

14. $\varepsilon=\mathrm{D} * 25 / 100 / / 25 \%$ as support threshold gave best results

15. $\mathrm{L} 1=\{$ frequent items with count $\geq \varepsilon\}$

16. for $\left(x=1 ; L_{x} \neq \varnothing ; x^{++}\right)$do begin

17. $\mathrm{C}_{\mathrm{x}+1}=$ candidate sets generated from $\mathrm{L}_{\mathrm{x}} / /$ Applying Join step

18. for each tuple $t$ in $D$ do

19. $\quad \mathrm{Mt}=\left\{\mathrm{c} \in \mathrm{C}_{\mathrm{x}+1} \mid \mathrm{c} \subseteq \mathrm{t}\right\}$

20. for candidates $\mathrm{c} \in \mathrm{Mt}$

21. $\quad \operatorname{count}[\mathrm{c}]=\operatorname{count}[\mathrm{c}]+1$

22. $\mathrm{L}_{\mathrm{x}+1}=\left\{\mathrm{c} \in \mathrm{C}_{\mathrm{x}+1} \mid \operatorname{count}[\mathrm{c}] \geq \varepsilon\right.$ 
23. end // for

\section{// Applying Prune step}

24. Generate ARS for $\cup_{x} L_{x}$

25. Calculate $\mathrm{cv}$ for each $\mathrm{AR}$ where $\mathrm{AR} \in \mathrm{ARS}$ as follows:

$$
\operatorname{cv}(A \rightarrow B)=\frac{\operatorname{Support}(A \cup B)}{\text { Support }(A)}
$$

26. Calculate iv for each AR where AR $\in$ ARS as follows:

$$
i v(A \rightarrow B)=\frac{\operatorname{Support}(A \cup B)}{\operatorname{Support}(A) * \operatorname{Support}(B)}
$$

27. $A=\{A R \in A R S \mid(\operatorname{cv}[A R] \geq$ min_conf $)$ and (iv $[A R] \geq$ min_imp) $\}$

28. Return A

\section{IMPLEMENTATION AND EXPERIMENTAL RESULTS}

The used medical dataset was especially collected following a selective and thoroughly method to guarantee that it is highly representative for real negative, positive mild, and positive severe COVID-19 patients' cases. It represents real 291 COVID-19 investigated patients (127 COVID-19 Negative Patients includes 68 Influenza patients and 59 normal cases, 84 COVID-19 Positive Mild patients, and 80 COVID-19
Positive Severe patients). Based on a review of practice, chest consultants' opinions surveys, and empirical literature, the used dataset covers the most important COVID-19 diagnosis parameters. $70 \%$ of the prepared dataset was used in training and $30 \%$ of the dataset is used in testing the mining models. The proposed hybrid model was implemented using SQL Server Analysis Services and Visual Studio IDE. In the following subsections, the results of implementing the proposed hybrid model.

\subsection{Proposed Soft Clustering mining model based on Scalable EM Results}

Fig. 3 presents the generated 10 soft clusters diagram for the COVID-19 investigated patients according to the Diagnosis Parameter with labels identifying the highest probability levels for each grade of the diagnosis parameter: Negative, Positive Mild, and Positive Severe. The color density shows the distribution of the learning dataset population according to diagnosis value among the identified soft clusters (Positive Mild state 1 in this diagram). The connections show the relevant clusters. The shading of the line that connects one cluster to another represents the strength of the similarity of the clusters.

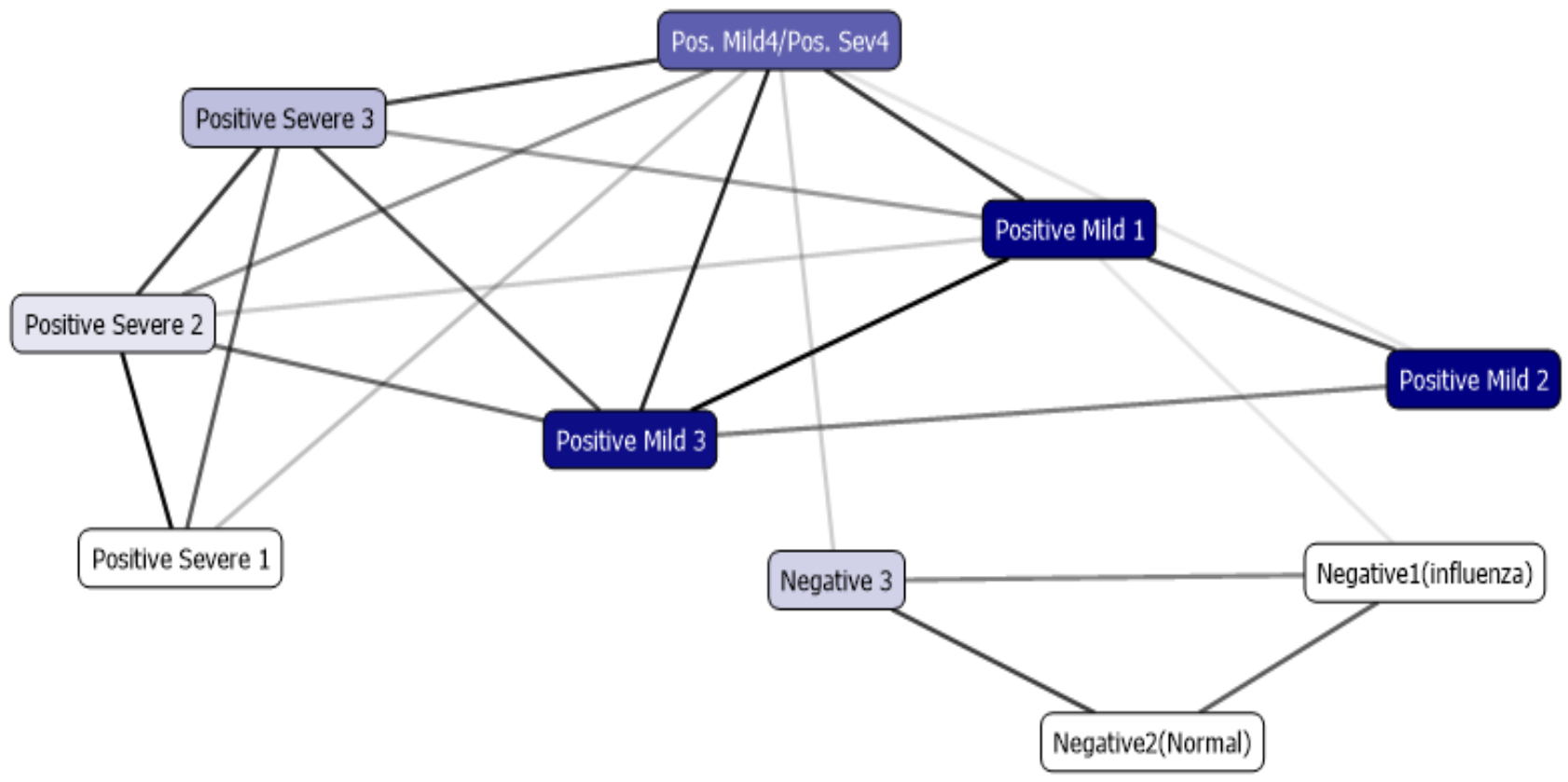

Fig. 3: Generated Soft Clusters Diagram from The Soft Clustering Mining Model (According to Positive Mild state 1 Distribution)

The implemented soft clustering mining model generated 10 clusters profiles for each one of all the COVID-19 diagnostic parameters and the diagnosis parameter with its states' distributions. Fig. 4 presents Part of the Generated COVID-19 Soft Clusters Profiles with States Distributions for each Variable. From Fig. 4, COVID-19 positive severe population distributions are found mainly in four clusters labeled Positive Severe 1, Positive Severe 2, and Positive Severe 3. COVID-19 positive mild population distributions are found also mainly in three clusters i.e., Positive Mild 1, Positive Mild 2, and Positive Mild 3, and so on. After reviewing the generated soft clusters, the importance of the 20 COVID-19 diagnostic parameters were confirmed in their strong relationships with the COVID-19 three grades diagnosis: Negative, Positive Mild, and Positive Severe. Fig. 5, Fig. 6, Fig. 7, and Fig. 8 present the main characteristics of the soft COVID-19 Negative1(Influenza) for patients' cases infected with Influenza and COVID-19 Negative 2(Normal) for normal cases, Positive Mild 1 and Positive Severe 1 with their associated probabilities. The attributes that the cluster contains are listed in the Variables column, and the state of the listed attribute is listed in the Values column. 


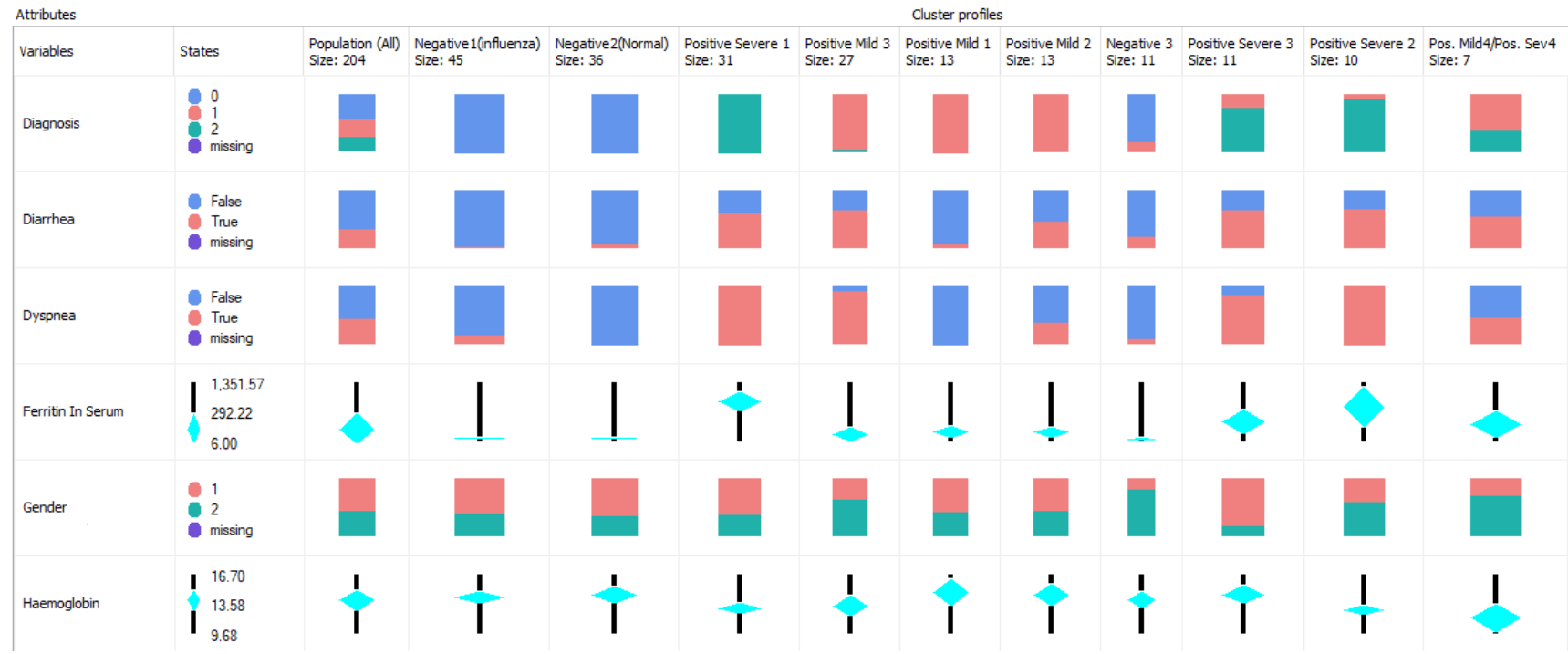

Fig. 4: Part of the Generated Soft Clusters Profiles with States Distributions for each Variable

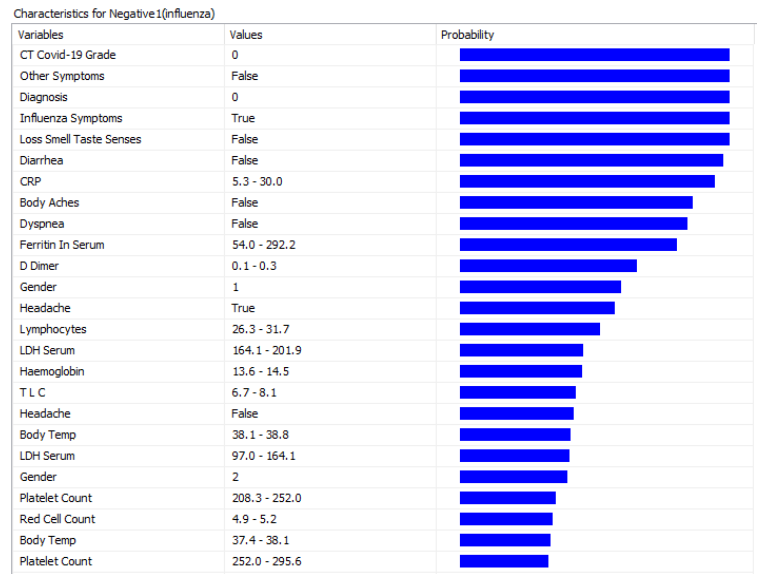

Fig. 5: Soft Cluster Negative 1 (Influenza) Characteristics

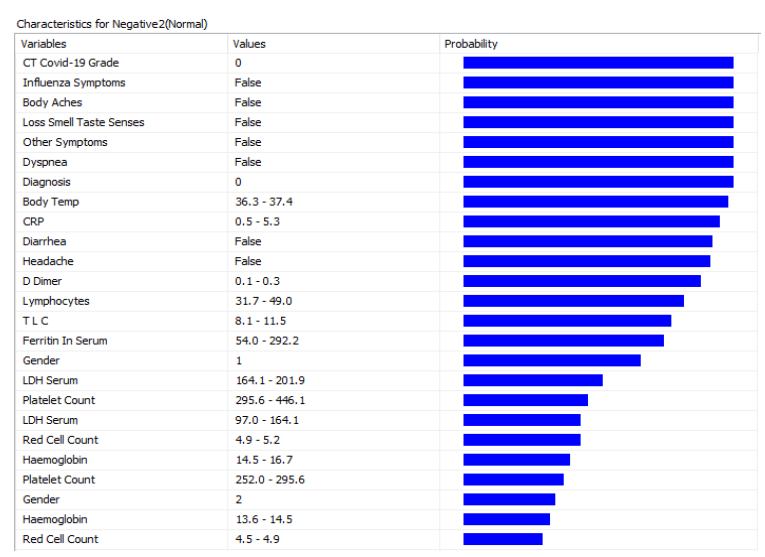

Fig. 6.: Soft Cluster Negative 2 (Normal) Characteristics

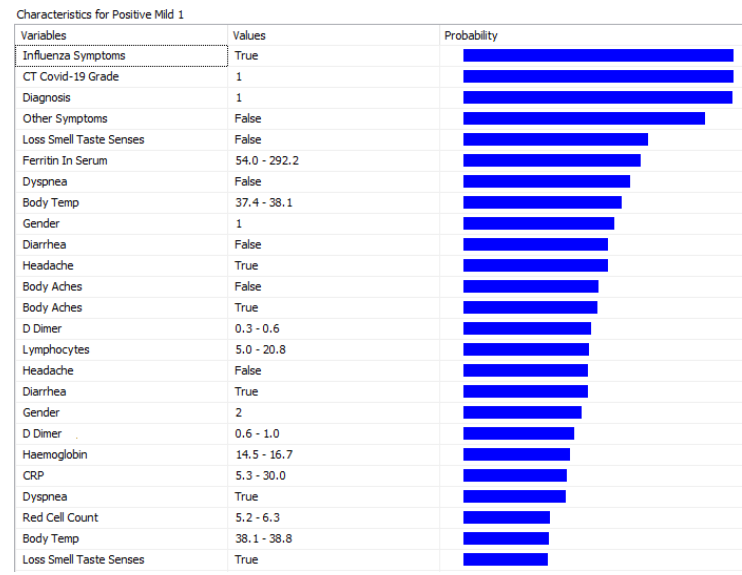

Fig. 7. Soft Cluster Positive Mild 1 Characteristics

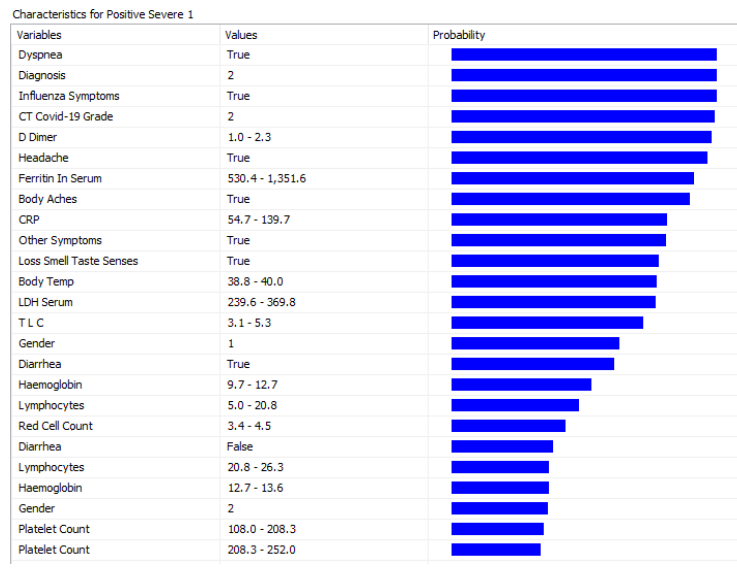

Fig. 8. Soft Cluster Positive Severe 1 Characteristics

The implemented soft clustering mining model successfully predicted accurately the relevant clusters for the different grades of COVID-19 diagnosis: Negative, Positive Mild, Positive Severe with their different states. 
4.2 Proposed Backpropagation based MLP Multiclass ANN Mining Model for COVID19 Diagnosis Prediction Results

After performing training and testing phases, the implemented proposed Backpropagation based MLP Multiclass ANN mining model for COVID-19 diagnosis prediction has generated very important results concerning the Negative, Positive Mild, and Positive Severe states (classes) for the diagnosis parameter. which are summarized in Table 4. Note that these results are related to the used relational dataset in training the model.

Table 4. Backpropagation based MLP ANN Mining Model for COVID-19 Diagnosis Prediction Important Results

\begin{tabular}{|c|c|c|c|c|}
\hline $\begin{array}{c}\text { Diagnosis } \\
\text { State } \\
\begin{array}{c}\text { Parameter } \\
\text { Value(s) }\end{array}\end{array}$ & $\begin{array}{c}\text { Covid- } \\
\text { Covid-19 } \\
\text { Negative } \\
\text { (Normal) }\end{array}$ & $\begin{array}{c}\mathbf{1 9} \\
\text { Negativ } \\
\mathbf{e} \\
\text { (Influen } \\
\text { za) }\end{array}$ & $\begin{array}{c}\text { Covid- } \\
\mathbf{1 9} \\
\text { Positiv } \\
\mathbf{e} \\
\text { Mild }\end{array}$ & $\begin{array}{c}\text { Covid- } \\
\mathbf{1 9} \\
\text { Positiv } \\
\mathbf{e} \\
\text { Severe }\end{array}$ \\
\hline (HB) & $13.5-16.7$ & $\begin{array}{c}13.5- \\
16.7\end{array}$ & $\begin{array}{c}13.5- \\
16.7\end{array}$ & $\begin{array}{c}9.95- \\
13.5\end{array}$ \\
\hline (RBC) & $4.8-6.3$ & $4.8-6.3$ & $4.8-$ & $3.4-$ \\
& & & 6.3 & 4.5 \\
\hline (PC) & $201-444$ & $201-444$ & $108-$ & $108-$ \\
& & & 290 & 290 \\
\hline (T.L.C.) & $7.9-11.0$ & $5.1-$ & $5.1-$ & $3.2-$ \\
& & 7.9 & 7.9 & 5.1 \\
\hline $\begin{array}{c}\text { Lymphocyte } \\
\text { S\% }\end{array}$ & $31.3-43$ & $20.5-$ & $3.9-$ & $3.9-$ \\
& & 31.3 & 20.5 & 20.5 \\
\hline $\begin{array}{c}\text { Ferritin in } \\
\text { serum (F) }\end{array}$ & $6-81$ & $81-331$ & $331-$ & $580-$ \\
\hline (LDH) & $97-166$ & $166-$ & $206-$ & $206-$ \\
serum & & 206 & 380 & 380 \\
\hline
\end{tabular}

\subsection{Proposed Improved Apriori Algorithm} based Association Rules (AR) mining model for COVID-19 Diagnosis Prediction Results

After performing training and testing phases, the implemented Proposed Improved Apriori Algorithm based Association Rules (AR) mining model for COVID-19 Diagnosis Prediction has generated the three dependency networks in Fig.9, Fig. 10, and Fig. 11 for the Negative, Positive Mild, and Positive severe states (classes) of the Diagnosis parameter.

\begin{tabular}{|c|c|c|c|c|}
\hline (CRP) & $0.5-7$ & $7-35$ & $35-64$ & $\begin{array}{l}64- \\
163\end{array}$ \\
\hline D Dimer & $0.1-0.29$ & $\begin{array}{c}0.29- \\
0.6\end{array}$ & $\begin{array}{c}0.689- \\
1.09\end{array}$ & $\begin{array}{c}1.09- \\
2.5\end{array}$ \\
\hline $\begin{array}{l}\text { CT COVID- } \\
19 \text { grade }\end{array}$ & 0 & 0 & 1 & 2 \\
\hline $\begin{array}{c}\text { Body Temp } \\
\text { (BT) }\end{array}$ & $\begin{array}{c}36.3- \\
37.4\end{array}$ & $\begin{array}{c}37.4- \\
38.1\end{array}$ & $\begin{array}{c}38.1- \\
38.9\end{array}$ & $\begin{array}{c}38.9- \\
40\end{array}$ \\
\hline Headache & $\mathrm{F}(0)$ & $\begin{array}{c}\mathrm{F}(0) / \\
\mathrm{T}(1)\end{array}$ & $\begin{array}{l}\mathrm{F}(0) / \\
\mathrm{T}(1)\end{array}$ & $\mathrm{T}(1)$ \\
\hline Body aches & $\mathrm{F}(0)$ & $\begin{array}{l}\mathrm{F}(0) / \\
\mathrm{T}(1)\end{array}$ & $\begin{array}{c}\mathrm{F}(0) / \\
\mathrm{T}(1)\end{array}$ & $\mathrm{T}(1)$ \\
\hline $\begin{array}{c}\text { Loss of } \\
\text { Smell \& } \\
\text { Taste senses }\end{array}$ & $\mathrm{F}(0)$ & $\mathrm{F}(0)$ & $\mathrm{T}(1)$ & $\mathrm{T}(1)$ \\
\hline Diarrhea & $\mathrm{F}(0)$ & $\mathrm{F}(0)$ & $\begin{array}{c}\mathrm{F}(0) / \\
\mathrm{T}(1)\end{array}$ & $\begin{array}{c}\mathrm{F}(0) / \\
\mathrm{T}(1)\end{array}$ \\
\hline Dyspnea & $\mathrm{F}(0)$ & $\mathrm{F}(0)$ & $\begin{array}{c}\mathrm{F}(0) / \\
\mathrm{T}(1)\end{array}$ & $\mathrm{T}(1)$ \\
\hline $\begin{array}{l}\text { Influenza } \\
\text { Like } \\
\text { Symptoms }\end{array}$ & $\bar{F}(0)$ & $\mathrm{T}(1)$ & $\mathrm{T}(1)$ & $\mathrm{T}(1)$ \\
\hline $\begin{array}{c}\text { Other } \\
\text { Symptoms }\end{array}$ & $\overline{F(0)}$ & $\mathrm{F}(0)$ & $\begin{array}{c}\mathrm{F}(0) / \\
\mathrm{T}(1)\end{array}$ & $\mathrm{T}(1)$ \\
\hline Gender & 1,2 & 1,2 & 1,2 & 1,2 \\
\hline Age & $9-74$ & $9-74$ & $4-80$ & $23-80$ \\
\hline
\end{tabular}

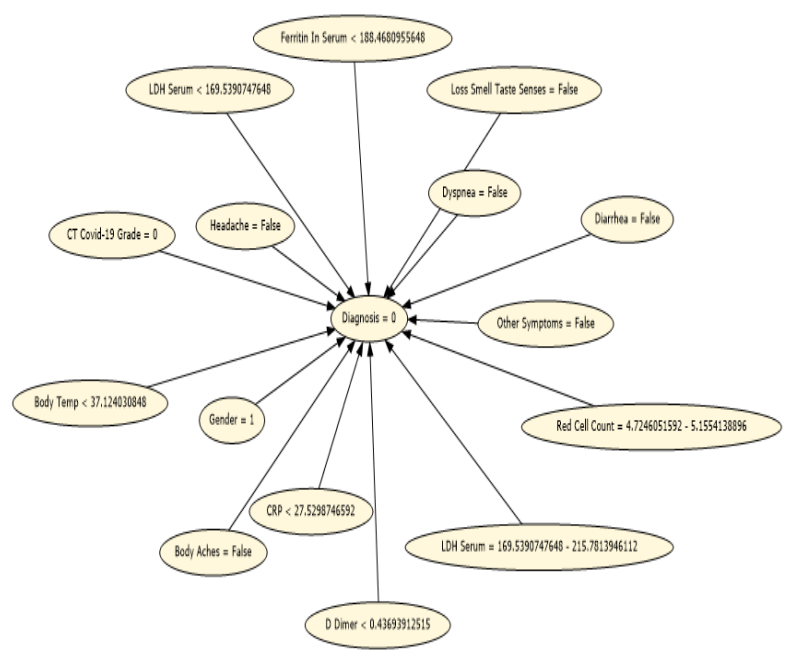

Fig. 9. COVID-19 Negative Dependency Network 


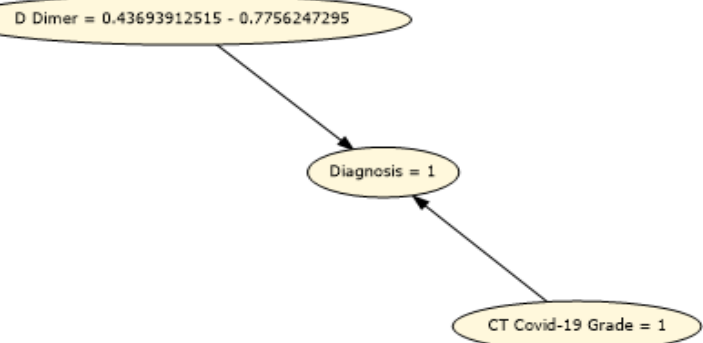

Fig. 10. COVID-19 Positive Mild Dependency Network

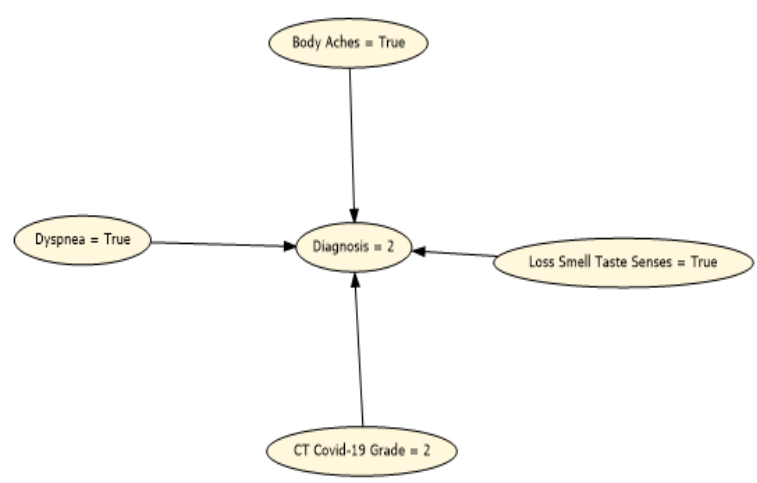

Fig. 11. COVID-19 Positive Severe Dependency Network

In addition, Fig. 12, Fig. 13, and Fig. 14 present the top generated association rules sorted in descending order according to importance related to diagnosing COVID-19 Negative (0), Positive Mild (1), and Positive Severe (3) states consecutively.

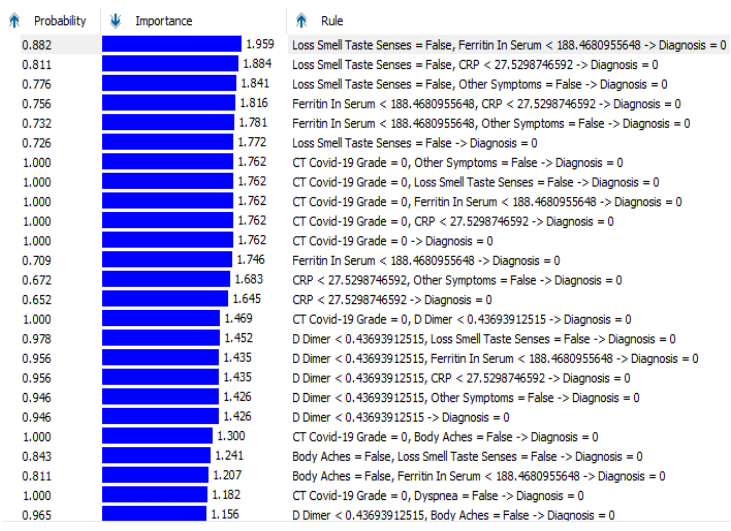

Fig. 12. COVID-19 Negative Association Rules

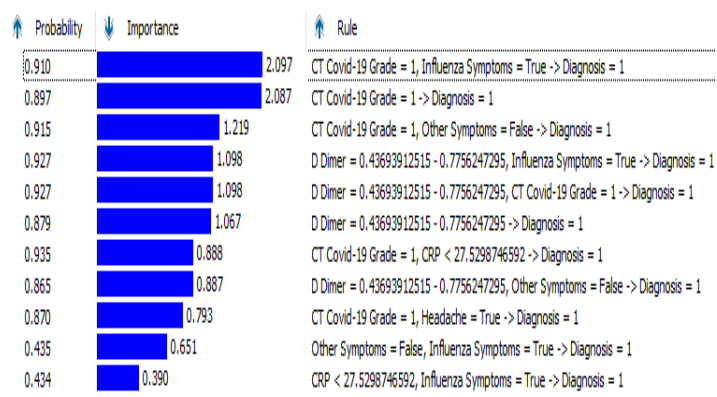

Fig. 13. COVID-19 Positive Mild Association Rules
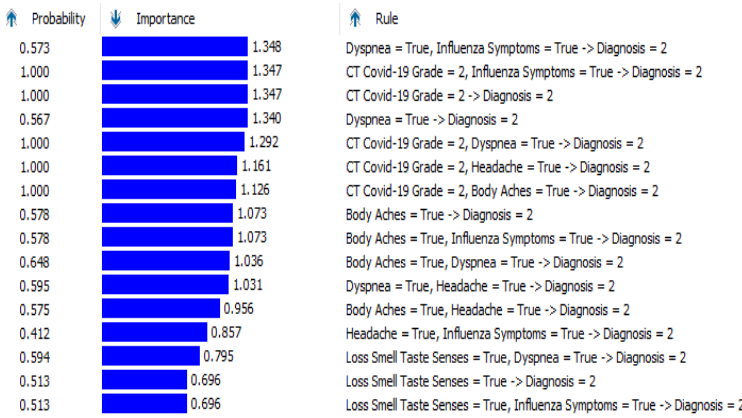

Fig. 14. COVID-19 Positive Severe Association Rules

\subsection{Proposed Hybrid Machine Learning Model for Predicting COVID-19 Disease Diagnosis Evaluation}

The implemented hybrid ML model was evaluated using different evaluation measures for the used predicting mining models. These used evaluation measures are derived from the generated confusion matrix related to each mining model which are presented in Table 5 and Table 6 . It must be noted that the built confusion matrix in our proposed ML model is for 3-class classification (multi-class classification problem) with 3 classes: Negative (0), Positive Mild(1), and Positive Severe(3) and generated for the testing dataset (87 data samples) which represents $30 \%$ of the total dataset. Unlike binary classification, there is no direct positive or negative classes here. As a result, in this case TP, TN, FP and FN for each individual class are calculated first, then each mining model accuracy, precision, recall(sensitivity), specificity, micro F1 score, and Matthews correlation coefficient (MCC) are calculated as follows: Accuracy formula is (6) listed in section 3.3

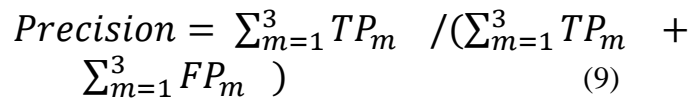

$$
\begin{aligned}
& \text { Recall(Sensitivity) }=\sum_{m=1}^{3} T P_{m} / \\
& \left(\sum_{m=1}^{3} T P_{m}+\sum_{m=1}^{3} F N_{m}\right) \\
& \text { (10) } \\
& \begin{array}{c}
\text { Specificity }=\sum_{m=1}^{3} T N_{m} \quad /\left(\sum_{m=1}^{3} T N_{m}+\right. \\
\left.\sum_{m=1}^{3} F P_{m}\right)
\end{array} \\
& \text { Micro F1 - score }=\frac{2 * \text { Total Recall } * \text { Total Precision }}{\text { Total Recall }+ \text { Total Precision }} \\
& \text { (12) } \\
& \text { Matthews correlation coefficient }(M C C)= \\
& \frac{\sum_{m=1}^{3} T P_{m} * \sum_{m=1}^{3} T N_{m}-\sum_{m=1}^{3} F P_{m} * \sum_{m=1}^{3} F N_{m}}{\sqrt{\left(\sum_{m=1}^{3} T P_{m}+\sum_{m=1}^{3} F P_{m}\right)\left(\sum_{m=1}^{3} T P_{m}+\sum_{m=1}^{3} F N_{m}\right)\left(\sum_{m=1}^{3} T N_{m}+\sum_{m=1}^{3} F P_{m}\right)\left(\sum_{m=1}^{3} T N_{m}+\sum_{m=1}^{3} F N_{m}\right)}}
\end{aligned}
$$

Where TP: True Positive; TN: True Negative; FP: False Positive; FN: False Negative, and m: It takes value from 1 to 3 representing three diagnosis classes (Negative(0), Positive Mild(1), Positive Severe(3)). 
Table 5. Confusion Matrix for Proposed Backpropagation based MLP ANN Mining Model

\begin{tabular}{|c|c|c|c|c|}
\hline Predicted & $\begin{array}{c}\mathbf{0} \\
\text { (Actual) }\end{array}$ & $\begin{array}{c}\mathbf{1} \\
\text { (Actual) }\end{array}$ & $\begin{array}{c}\mathbf{2} \\
\text { (Actual) }\end{array}$ & Sum \\
\hline 0 & 37 & 1 & 0 & 38 \\
\hline 1 & 1 & 26 & 0 & 27 \\
\hline 2 & 1 & 1 & 20 & 22 \\
Sum & 39 & 28 & 20 & \\
\hline
\end{tabular}

Table 6. Confusion Matrix for Proposed Improved Apriori Algorithm based Association Rules (AR) Mining Model

\begin{tabular}{|c|c|c|c|c|}
\hline Predicted & $\begin{array}{c}\mathbf{0} \\
\text { (Actual) }\end{array}$ & $\begin{array}{c}\mathbf{1} \\
\text { (Actual) }\end{array}$ & $\begin{array}{c}\mathbf{2} \\
\text { (Actual) }\end{array}$ & Sum \\
\hline 0 & 37 & 2 & 0 & 39 \\
\hline 1 & 0 & 21 & 1 & 22 \\
\hline 2 & 0 & 0 & 26 & 26 \\
\hline Sum & 37 & 23 & 27 & \\
\hline
\end{tabular}

Table. 7 presents the calculated evaluation measures for the proposed two COVID-19 prediction mining models, and the evaluation measures average for the proposed hybrid machine learning model as a whole. From Table. 7 we can conclude that the proposed hybrid machine learning model has successfully not only classified COVID-19 patients into positive mild and positive severe patients, but also discriminated successfully between COVID-19 patients and Influenza patients/normal cases with an average accuracy of $97.3 \%$, an average precision $96 \%$, an average recall (sensitivity) $96 \%$, an average specificity $98 \%$, and average micro F1 score $96 \%$ on an independent testing dataset of 87 patients. Unlike the other evaluation metrics, MCC takes all the cells of the Confusion Matrix into consideration in its formula. The range of values of MCC lie between -1 to +1 . A model with a score of +1 is a perfect model and -1 is a poor model. The average MCC of the hybrid model is 0.94 which means that the proposed model achieved a high degree of perfection.

Table. 8 and Fig. 15 show a comparison between the proposed model and the state-of-the art models regarding to the model accuracy, sensitivity, and specificity. The results show that the proposed model outperforms the other reviewed COVID-19 diagnosis prediction models.

Table 7. The Proposed Hybrid Machine Learning Evaluation Measures

\begin{tabular}{|l|c|c|c|c|c|c|}
\hline Measure & Accuracy & Precision & Recall/ & Specificity & $\begin{array}{c}\text { Micro } \\
\text { F1 } \\
\text { Score } \\
\text { Matthews } \\
\text { correlation } \\
\text { coefficient } \\
\text { (MCC) }\end{array}$ \\
\hline $\begin{array}{l}\text { Backpropagation based MLP ANN Mining } \\
\text { Model for COVID-19 Diagnosis Prediction }\end{array}$ & $96.9 \%$ & $95.4 \%$ & $95.4 \%$ & $97.7 \%$ & $95.4 \%$ & 0.93 \\
\hline $\begin{array}{l}\text { Association Rules mining model based on } \\
\text { Improved Apriori algorithm for COVID-19 } \\
\text { Diagnosis Prediction }\end{array}$ & $97.7 \%$ & $96.6 \%$ & $96.6 \%$ & $98.3 \%$ & $96.6 \%$ & 0.95 \\
\hline $\begin{array}{l}\text { The proposed hybrid machine learning model } \\
\text { evaluation measures Average }\end{array}$ & $97.3 \%$ & $96 \%$ & $96 \%$ & $98 \%$ & $96 \%$ & 0.94 \\
\hline
\end{tabular}

Table 8. COVID-19 Diagnosis Models Evaluation Measures Comparison

\begin{tabular}{|c|c|c|c|}
\hline & Accuracy\% & sensitivity\% & specificity\% \\
\hline COVID-19 Diagnosis proposed Model & 97.3 & 96 & 98 \\
\hline Banerjee et al. Model & 87.00 & 65.00 & 80.00 \\
\hline Bao et al. Model & 84.00 & 88.00 & 93.60 \\
\hline Barbosa et al. Model & 95.20 & 96.80 & 95.00 \\
\hline Nan et al. Model & 91.00 & 87.00 & \\
\hline
\end{tabular}




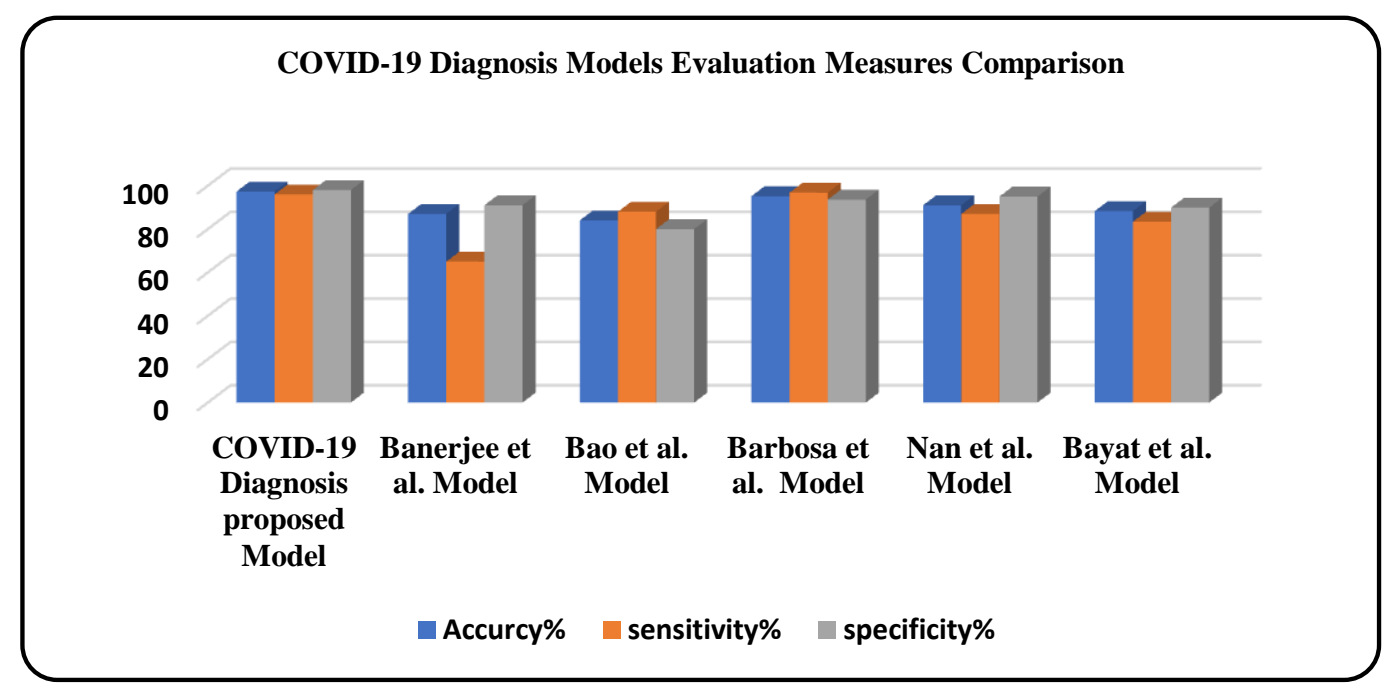

Fig. 15. COVID-19 Diagnosis Models Evaluation Measures Comparison Graph

\section{CONCLUSION AND FUTURE WORK}

In this study, a hybrid two-phase machine learning model for COVID-19 diagnosis prediction was proposed. The aim of this proposed model is to early diagnose COVID-19 based on available laboratory tests results, clinical symptoms, CT results, and demographic data in case of the difficulty of applying or absence of PCR test. The dataset consisted of 291 data samples (127 COVID-19 Negative Patients include 68 Influenza patients and 59 normal cases, 84 COVID-19 confirmed Positive Mild patients, and 80 COVID-19 confirmed Positive Severe patients). The soft clustering mining model was able to successfully identify the most relevant identifying clusters characteristics for the COVID-19 disease grades and confirmed the selected 20 features strong relationships with the COVID-19 three grades diagnosis: Negative, Positive Mild, and Positive Severe. The implementation of the two proposed ML mining models for COVID-19 diagnosis achieved an overall accuracy 97.3\%, sensitivity $96 \%$, and specificity $98 \%$. These achieved results outperformed the other reviewed state-of-the art COVID-19 diagnosis prediction models, taking into consideration the proposed unique features combination used efficiently for COVID-19 diagnosis in his study. Future work will focus on integrating this proposed hybrid ML model with an intelligent COVID-19 treatment protocols advisor system according to the disease grade, patient history, clinical signs and symptoms, and physical examination.

\section{REFERENCES}

[1] Sohrabi, C., AlsafiZ OfiNeill, N., Khan, M. and Kerwan A. et al. 2020. World health organization declares global emergency: a review of the 2019 novel coronavirus (COVID-19). International Journal of Surgery. vol. 76, 71-76.

[2] Barbat, MM., Wesche, C., Werhli, AV., and Mata, MM. 2019. An adaptive machine learning approach to improve automatic iceberg detection from SAR images . ISPRS Journal of Photogrammetry and Remote Sensing. vol. 156, 247-259.

[3] Shang, R., Qi, L., Jiao, L., Stolkin, R., and Li, Y. 2014. Change detection in SAR images by artificial immune multi-objective clustering. Engineering Applications of Artificial Intelligence. vol. 31, 53-67.

[4] Choi, S., Kang, M-G., Min, H., Chang, Y-S., and Yoon,
S. 2017. Large-scale machine learning of media outlets for understanding public reactions to nation-wide viral infection outbreaks. Methods. vol. 129, 50-59.

[5] Vaka, AR., and Soni, B. 2020. Breast cancer detection by leveraging Machine Learning. ICT Express. vol. 6. no. 4, 320-324.

[6] Saxena, S., and Gyanchandani, M. 2019. Machine learning methods for computer-aided breast cancer diagnosis using histopathology: a narrative review. Journal of Medical Imaging and Radiation Science. vol. 51. no. 1, 182-193.

[7] Vaishya, R., Javaid, M., Khan, IH., and Haleem, A. 2020. Artificial Intelligence (AI) applications for COVID-19 pandemic. Diabetes \& Metabolic Syndrome: Clinical Research \& Reviews. vol.14. no. 4, 337-339.

[8] T. Ai, Z. Yang, H. Hou, C. Zhan, C. Chen, W. Lv, Q. Tao, Z. Sun, and L. Xia, "Correlation of chest CT and RT-PCR testing in coronavirus disease 2019 (COVID19) in China: a report of 1014 cases", Radiology, vol. 296, no. 2, pp. 32-40, 2020.

[9] Javor, D., Kaplan, A., Puchner, S.B., Krestan, C., and Baltzer, P. 2020. Deep learning analysis provides accurate COVID-19 diagnosis on chest computed tomography. European Journal of Radiology. vol. 133. no. 109402, 2020.

[10] Goodman-Meza, D., Adamson, PC., and Ebinger, J. et al. 2020. A machine learning algorithm to increase COVID19 inpatient diagnostic capacity. PLOS ONE Journal. vol. 15. no. 9, e0239474.

[11] An, C., Lim, H., and Kim, DW. et al. 2020. Machine learning prediction for mortality of patients diagnosed with COVID-19: a nationwide Korean cohort study . Scientific Reports Journal. Vol. 10. no. 18716.

[12] Khanday, A.M.U.D., Rabani, S.T., and Khan, Q.R., et al. 2020. Machine learning based approaches for detecting COVID-19 using clinical text data. Int. Journal of Information Technology. vol. 12. 731-739.

[13] Banerjee, A., Ray, S., Vorselaars, B., and Kitson, J. et al. 2020. Use of Machine Learning and Artificial Intelligence to predict SARS-CoV-2 infection from Full Blood Counts in a population. Int. 
immunopharmacology. vol. 86. no. 106705.

[14] Bao, FS., He, Y., Liu, J., Chen, Y., Li, Q., and Zhang, CR. et al. 2020. Triaging moderate covid-19 and other viral pneumonias from routine blood tests. arXiv eprints. arXiv:2005.06546.

[15] Barbosa, V., Gomes, JC., Santana, MA., and Almeida Albuquerque, JE. et al. Heg. ia: an intelligent system to support diagnosis of covid-19 based on blood tests. medRxiv, 2020.

[16] Nan, SN., Ya, Y., Ling, TL., and Nv, GH., et al. A prediction model based on machine learning for diagnosing the early covid-19 patients. medRxiv, 2020.

[17] Bayat, V., Phelps, S., and Ryono, R., et al. A Severe Acute Respiratory Syndrome Coronavirus 2 (SARS-
CoV-2) Prediction Model from Standard Laboratory Tests, Clinical Infectious Diseases. ciaa1175. 2020.

[18] Zoabi, Y., Deri-Rozov, S., and Shomron, N. 2021. Machine learning-based prediction of COVID-19 diagnosis based on symptoms. npj Digital Medical. vol. 4. no. 3 .

[19] Prokop, M., van Everdingen, W., and Van Rees, VT. et al. 2020. CO- RADS - a categorical CT assessment scheme for patients with suspected COVID- 19: definition and evaluation. Radiology. vol. 296. no. 2, 97 104.

[20] Bradley P., Fayyad, U., and Reina, C. 1998. Scaling EM (Expectation-Maximization) Clustering to Large Databases. MSR-TR-98-3.1998. 